\title{
A NOTE ON TORSION-FREE ABELIAN GROUPS OF FINITE RANK
}

\author{
W. WICKLESS AND C. VINSONHALER
}

Abstract. Let $G$ be a torsion-free abelian group of rank $n$ and $X=\left\{x_{1}, \cdots, x_{n}\right\}$ a maximal set of rationally independent elements in $G$. It is well known that any $g \in G$ can be uniquely written $g=$ $\alpha_{1} x_{1}+\cdots+\alpha_{n} x_{n}$, for some $\alpha_{1}, \cdots, \alpha_{n} \in Q$, the rational numbers. This enables us to define, for any such $(G, X)$, a collection of subgroups of $Q$ and "natural" isomorphisms, denoted by $S(G, X)$. It is known that if $G$ is of rank two, then $G$ may be recovered from $S(G, X)$ is a natural way. The following result is obtained for groups of rank greater than two:

THEOREM. Let $G, G^{\prime}$ be torsion free abelian groups of finite rank with $S(G, X)=S\left(G^{\prime}, X^{\prime}\right)$ for suitable $X, X^{\prime}$. Let $F, F^{\prime}$ be the free subgroups of $G, G^{\prime}$ generated by $X, X^{\prime}$. Then $G\left|F \cong G^{\prime}\right| F^{\prime}$.

An additional condition is given for pairs $(G, X),\left(G^{\prime}, X^{\prime}\right)$ such that $S(G, X)=S\left(G^{\prime}, X^{\prime}\right)$ implies $G \cong G^{\prime}$.

1. Schemes of groups. Let $G$ be a group ${ }^{1}$ of rank $n$ and $X=\left\{x_{1}, \cdots, x_{n}\right\}$ a maximal set of rationally independent elements in $G$. It is well known that any $g \in G$ can be uniquely written $g=\alpha_{1} x_{1}+\cdots+\alpha_{n} x_{n}$, for some $\alpha_{1}, \cdots, \alpha_{n} \in Q$, the rational numbers. This enables us to define, for any such $(G, X)$, a collection of subgroups of $Q$ as follows:

DEFINITION 1. Let $1 \leqq i \leqq n$ and $i \notin J \subset\{1,2, \cdots, n\}$. Then

$$
\begin{aligned}
A_{J}^{i} & =A_{J}^{i}(G, X) \\
& =\left\{\alpha \in Q \mid \alpha_{1} x_{1}+\cdots+\alpha_{i-1} x_{i-1}+\alpha x_{i}+\alpha_{i+1} x_{i+1}+\cdots+\alpha_{n} x_{n} \in G\right. \\
& \left.\quad \text { for some set of } \alpha_{j} \in Q \text { with } \alpha_{j}=0 \quad \forall j \in J\right\} .
\end{aligned}
$$

The $A_{J}^{i}$ are clearly subgroups of the additive rationals and contain the integers, $Z$. Furthermore, $A_{J}^{i} \supseteq A_{J^{\prime}}^{i}$ if $J \subseteq J^{\prime}$. For convenience, we write $A^{i}=A_{\phi}^{i}$. The $A_{J}^{i}$ can be used to define a collection of "natural" isomorphisms.

Lemma 1. Given $1 \leqq i \neq j \leqq n$, with $i, j \notin J \subset\{1,2, \cdots, n\}$, there exists an isomorphism

$$
A_{J}^{i} / A_{\{j\} \cup J}^{i} \stackrel{\theta}{\cong} A_{J}^{j} / A_{\{i\} \cup J}^{j}
$$

Presented to the Society, September 1, 1972; received by the editors March 20, 1972. AMS (MOS) subject classifications (1970). Primary 20K15.

1 We write "group" for torsion-free abelian group throughout.

(C) American Mathematical Society 1973 
Proof. Let $\alpha_{i} \in A_{J}^{i}$ and write $\sum_{k=1}^{n} \alpha_{k} x_{k} \in G$ for some choice of $\alpha_{k} \in Q$, $k \neq i$, with $\alpha_{k}=0$ for $k \in J$. Then $\left(\alpha_{i}+A_{\{j\} \cup J}^{i}\right)^{\theta}=\alpha_{j}+A_{\{i\} \cup J}^{j}$ is the required isomorphism.

We call the collection of groups $A_{J}^{i}$ and isomorphisms $\theta$ the scheme of $(G, X)$, and denote it by $S(G, X)$.

Lemma 2. Given $1 \leqq i, j, k \leqq n$ with $i, j, k \notin J \subset\{1, \cdots, n\}$ and $i, j, k$ distinct, then

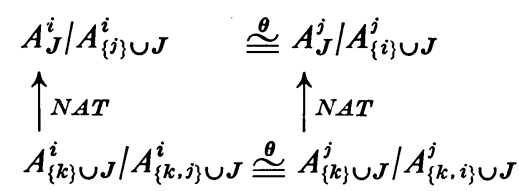

is a commutative diagram where the $\theta$ 's are the isomorphisms of Lemma 1 and NAT is the composition of the obvious inclusion and factor maps.

Proof. The proof follows immediately from the definition of the maps $\theta$.

If $G$ has rank 2, Beaumont and Wisner [2] showed that $G$ may be recovered from the groups $A_{J}^{i}$ and isomorphism $\theta$ in the following way: Let $x_{1}, x_{2}$ be independent elements in $G$ and $\left\{A^{1}, A_{2}^{1}, A^{2}, A_{1}^{2}, \theta\right\}$ the scheme of $\left(G,\left\{x_{1}, x_{2}\right\}\right)$. Then $G^{\prime}=\left\{(\alpha, \beta) \mid \alpha \in A_{1},\left(\alpha+A_{2}^{1}\right)^{\theta}=\beta+A_{1}^{2}\right\}$ is isomorphic to $G$. Moreover, $S\left(G^{\prime},\{(1,0),(0,1)\}\right)=S\left(G,\left\{x_{1}, x_{2}\right\}\right)$.

The question then arises: To what extent does $S(G, X)$ determine $G$ in general? The results which follow give a partial answer.

Since any group $G$ of rank $n$ with maximal independent set $X=$ $\left\{x_{1}, \cdots, x_{n}\right\}$ can be identified with a subgroup of $Q^{n}$ under the map $\alpha_{1} x_{1}+\cdots+\alpha_{n} x_{n} \rightarrow\left(\alpha_{1}, \cdots, \alpha_{n}\right)$, it suffices to consider pairs $(G, X)$ with $Z^{n} \subseteq G \subseteq Q^{n}$ and $X=\{(1,0,0, \cdots, 0),(0,1,0, \cdots, 0), \cdots,(0,0, \cdots, 0,1)\}$. Let $(G, X)$ and $\left(G^{\prime}, X\right)$ be two such pairs.

Lemma 3. If $S(G, X)=S\left(G^{\prime}, X\right)$ then given any element $\left(\alpha_{1}, \alpha_{2}, \cdots, \alpha_{n}\right) \in$ $G$ and fixed integers $i \neq j$, with $1 \leqq i, j \leqq n$, there exists an element $\left(\alpha_{1}^{\prime}, \alpha_{2}^{\prime}, \cdots, \alpha_{n}^{\prime}\right) \in G^{\prime}$ with $\alpha_{i}=\alpha_{i}^{\prime}, \alpha_{j}=\alpha_{j}^{\prime}$. Moreover, it is possible to choose $\alpha_{k}^{\prime}=0$ whenever $\alpha_{k}=0$.

Proof. By symmetry, we may take $i=1, j=2$. Let $K=\left\{k \mid \alpha_{k}=0\right\}$. Since $\alpha_{1} \in A_{K}^{1}$ and $A_{K}^{1}(G, X)=A_{K}^{1}\left(G^{\prime}, X\right)$, there exists $\left(\alpha_{1}, \beta_{2}, \beta_{3}, \cdots, \beta_{n}\right) \in G^{\prime}$ with $\beta_{k}=0$ for $k \in K$. Thus, $\beta_{2}+A_{\{1\} \cup K}^{2}=\left[\alpha_{1}+A_{\{2\} \cup K}^{1}\right]^{\theta}=\alpha_{2}+A_{\{1\} \cup K}^{2}$. Hence, $\alpha_{2}-\beta_{2} \in A_{\{1\} \cup K}^{2}$, and there exists $\left(0, \alpha_{2}-\beta_{2}, \gamma_{3}, \gamma_{4}, \cdots, \gamma_{n}\right) \in G^{\prime}$ with $\gamma_{k}=0$ for $k \in K$. Then $\left(\alpha_{1}, \alpha_{2}, \alpha_{3}^{\prime}, \cdots, \alpha_{n}^{\prime}\right)=\left(0, \alpha_{2}-\beta_{2}, \gamma_{3}, \cdots, \gamma_{n}\right)+$ $\left(\alpha_{1}, \beta_{2}, \cdots, \beta_{n}\right) \in G^{\prime}$ with $\alpha_{k}^{\prime}=0$ for $k \in K$. 
Lemma 4. Let $G, G^{\prime}, X$ be as in Lemma 3, and $p$ a prime integer. Let $g=\left(\alpha_{1}, \cdots, \alpha_{n}\right) \in G$ and $g_{1}^{\prime}, \cdots, g_{t}^{\prime} \in G^{\prime}$ be such that $\bar{g}=g+Z^{n}, \bar{g}_{1}^{\prime}=$ $g_{1}^{\prime}+Z^{n}, \cdots, \bar{g}_{t}^{\prime}=g_{t}^{\prime}+Z^{n}$ are independent elements in the p-primary component of $Q^{n} / Z^{n}$. Then there exists $g^{\prime}=\left(\alpha_{1}^{\prime}, \cdots, \alpha_{n}^{\prime}\right) \in G^{\prime}$, with $\operatorname{order}\left(\bar{g}^{\prime}\right) \geqq$ $\operatorname{order}(\bar{g})=p^{k}$, such that $\bar{g}_{1}^{\prime}, \cdots, \bar{g}_{t}^{\prime}, \bar{g}^{\prime}$ are independent. If $\alpha_{r}=a_{r} / p^{k}$, $\alpha_{s}=a_{s} / p^{k^{\prime}}$, for integers $a_{r}, a_{s}$ with $\left(a_{r}, p\right)=\left(a_{s}, p\right)=1, k^{\prime} \leqq k$, then $g^{\prime}$ can be chosen so that $c \alpha_{r}=\alpha_{r}^{\prime}, c \alpha_{s}=\alpha_{s}^{\prime}$, where $c$ is an integer with $(c, p)=1$. Furthermore, we can choose $\alpha_{i}^{\prime}=0$ whenever $\alpha_{i}=0$.

Proof. We proceed by induction on the number, $m$, of nonzero coordinates of $g$. If $m=1,2$, then $g \in G^{\prime}$ by Lemma 3 and the result follows. Suppose now that $g=\left(\alpha_{1}, \cdots, \alpha_{m}, 0,0, \cdots, 0\right)$. We assume, without loss of generality, that all integral components of $g$ are zero, and, by renumbering if necessary, that the nonzero coordinates appear first. Write $\alpha_{i}=a_{i} \mid p^{k_{i}}, 1 \leqq k_{i} \leqq k$, where $\left(a_{i}, p\right)=1$. Again by renumbering, assume $r=1, s=2$. Thus, $k_{1}=k$. By Lemma 3 , there exists

$$
h_{1}=\left(a_{1} / p^{k_{1}}, a_{2} / p^{k_{2}}, \beta_{3}, \cdots, \beta_{m}, 0, \cdots, 0\right) \in G^{\prime} \text {. }
$$

Choose an integer $c$ with $(c, p)=1$ such that

$$
h_{2} \equiv c h_{1}=\left(c a_{1} / p^{k_{1}}, c a_{2} / p^{k_{2}}, b_{3} / p^{l_{3}}, \cdots, b_{m} / p^{l_{m}}, 0, \cdots, 0\right) .
$$

Note that $\left\{\bar{g}_{1}^{\prime}, \cdots, \bar{g}_{t}^{\prime}, c \bar{g}\right\}$ is still independent. (The integer $c$ times a power of $p$ is divisible by the denominators of $\beta_{3} \cdots \beta_{m}$.)

Case I. Suppose $l_{i}=k_{i}$ and $p \mid c a_{i}-b_{i}$ for $3 \leqq i \leqq m$. Then $\operatorname{order}\left(h_{2}\right)=p^{k}$. We show $\left\{\bar{g}_{1}^{\prime}, \cdots, \bar{g}_{t}^{\prime}, \bar{h}_{2}\right\}$ is independent. It suffices to show $\left\{p^{j_{1}-1} \bar{g}_{1}^{\prime}, \cdots\right.$, $\left.p^{j_{t}-1} \bar{g}_{t}^{\prime}, p^{k-1} \bar{h}_{2}\right\}$ is independent in $\bar{G}^{\prime}[p]$, where order $\bar{g}_{i}^{\prime}=p^{j_{i}}$. Suppose $u_{1} p^{j_{1}-1} \bar{g}_{1}^{\prime}+\cdots+u_{t} p^{j_{t}-1} \bar{g}_{t}^{\prime}+u p^{k-1} \bar{h}_{2}=0$ with $(p, u)=1$. Clearly, $u_{1} p^{j_{1}-1} \bar{g}_{1}^{\prime}+$ $\cdots+u_{t} p^{j_{t}-1} \bar{g}_{t}^{\prime}+u p^{k-1} c \bar{g} \neq 0$. Subtraction yields $u p^{k-1}\left(c \bar{g}-\bar{h}_{2}\right) \neq 0$. But, since $p \mid c a_{i}-b_{i}, i=3,4, \cdots, m, u p^{k-1}\left(c \bar{g}-\bar{h}_{2}\right)=0$, a contradiction. Thus, in Case I, $g^{\prime}=h_{2}$ is the desired element of $G^{\prime}$.

Case II. Assume without loss of generality that $l_{3} \neq k_{3}$, or that $p \nmid c a_{3}-b_{3}$. It is then easy to show that $1 / p^{k_{3}} \in A_{\{1, m+1, m+2, \cdots, n\}}^{3}$. Thus there exist $\Gamma_{1}=\left(0, x, c a_{3} / p^{k_{3}}, x_{4}, \cdots, x_{m}, 0,0, \cdots, 0\right) \in G$ and (by Lemma 3 ) $\Gamma_{1}^{\prime}=\left(0, x, c a_{3} / p^{k_{3}}, x_{4}^{\prime}, \cdots, x_{m}^{\prime}, 0, \cdots, 0\right) \in G^{\prime}$. Applying Lemma 3 again to $\Gamma_{2}=c g-\Gamma_{1}$, we have

$$
\Gamma_{2}^{\prime}=\left(c a_{1} / p^{k_{1}}, c a_{2} / p^{k_{2}}-x, 0, y_{4}^{\prime}, \cdots, y_{m}^{\prime}, 0,0, \cdots, 0\right) \in G^{\prime} .
$$

Consider $h_{2}-\Gamma_{1}^{\prime}-\Gamma_{2}^{\prime}=\left(0,0, b_{3} / p^{l_{3}}-c a_{3} / p^{k_{3}}, z_{4}^{\prime}, \cdots, z_{m}^{\prime}, 0,0, \cdots, 0\right)$. Since $l_{3} \neq k_{3}$ or $p \nmid b_{3}-c a_{3}$, it follows that $1 / p^{k_{3}} \in A_{\{1,2, m+1, m+2, \cdots, n\}}^{3}$. Let

and

$$
\Gamma_{3}=\left(0,0, c a_{3} / p^{k_{3}}, v_{4}, \cdots, v_{m}, 0,0, \cdots, 0\right) \in G
$$

$$
\Gamma_{3}^{\prime}=\left(0,0, c a_{3} / p^{k_{3}}, v_{4}^{\prime}, \cdots, v_{m}^{\prime}, 0,0, \cdots, 0\right) \in G^{\prime}
$$


Finally, let

$$
\Gamma_{4}^{\prime}=\Gamma_{1}^{\prime}+\Gamma_{2}^{\prime}-\Gamma_{3}^{\prime}=\left(c a_{1} / p^{k_{1}}, c a_{2} / p^{k_{2}}, 0, w_{4}^{\prime}, \cdots, w_{m}^{\prime}, 0,0, \cdots, 0\right) .
$$

If $\left\{\bar{g}_{1}^{\prime}, \cdots, \bar{g}_{t}^{\prime}, \Gamma_{4}^{\prime}\right\}$ is independent, it is easy to construct the desired element $g^{\prime} \in G^{\prime}$. If $\left\{\bar{g}_{1}^{\prime}, \cdots, \bar{g}_{t}^{\prime}, \overline{c g-\Gamma_{3}}\right\}$ is independent, the result follows from the induction hypothesis, after multiplication by a suitable integer prime to $p$.

Otherwise, $\Gamma_{4}^{\prime}$ is dependent on, and $\Gamma_{3}$ is independent of the set $\left\{\bar{g}_{1}^{\prime}, \cdots, \bar{g}_{t}^{\prime}\right\}$. Multiplying $\Gamma_{3}$ by a suitable integer $d$, prime to $p$, and applying the induction hypothesis, we obtain

$$
\Gamma_{3}^{\prime \prime}=\left(0,0, c d a_{3} / p^{k_{3}}, v_{4}^{\prime \prime}, \cdots, v_{m}^{\prime \prime}, 0,0, \cdots, 0\right) \in G^{\prime}
$$

independent of $\bar{g}_{1}^{\prime}, \cdots, \bar{g}_{t}^{\prime}$, Now $\Gamma_{3}^{\prime \prime}+d \Gamma_{4}^{\prime}$ is still independent, and we can choose $e$ prime to $p$ such that $g^{\prime}=e\left(\Gamma_{3}^{\prime \prime}+d \Gamma_{4}^{\prime}\right)$ is the desired element.

Lemma 5. Let $G, G^{\prime}, X$ be as in Lemma 3. Let $\bar{g}_{1}, \cdots, \bar{g}_{t}$ be independent elements in the p-primary component of $\bar{G}$. Then there exist $\bar{g}_{1}^{\prime}, \cdots, \bar{g}_{t}^{\prime}$ independent in the p-primary component of $\bar{G}^{\prime}$ with $\operatorname{order}\left(\bar{g}_{i}^{\prime}\right) \geqq \operatorname{order}\left(\bar{g}_{i}\right)$.

Proof. The result follows by using induction on $t$ and applying Lemma 4. We now prove the main theorem.

THEOREM A. Let $G, G^{\prime}$ be groups of rank $n$ with maximal independent sets $Y, Y^{\prime}$ respectively, such that $S(G, Y)=S\left(G^{\prime}, Y^{\prime}\right)$. Let $F, F^{\prime}$ be the free subgroups of $G, G^{\prime}$ generated by $Y, Y^{\prime}$ respectively. Then $G\left|F \cong G^{\prime}\right| F^{\prime}$.

Proof. As before, we identify $G$ and $G^{\prime}$ with subgroups of $Q^{n}$ containing $Z^{n}$, such that $Y$ and $Y^{\prime}$ are identified with the set $X$ of standard basis vectors. Write

$$
G / Z^{n}=\sum \oplus A_{p}, \quad G^{\prime} / Z^{n}=\sum \oplus A_{p}^{\prime}
$$

where $A_{p}$ and $A_{p}^{\prime}$ are the respective $p$-primary components. Note that $A_{p}$ and $A_{p}^{\prime}$ are subgroups of $\left(Q^{n} / Z^{n}\right)_{p}$, and therefore

$$
\begin{gathered}
A_{p}=\sum_{i=1}^{r} \oplus Z\left(p^{k_{i}}\right), \\
A_{p}^{\prime}=\sum_{i=1}^{r^{\prime}} \oplus Z\left(p^{k_{i}^{\prime}}\right) \quad \text { where } 1 \leqq k_{i} \leqq \infty, \quad 1 \leqq k_{i}^{\prime} \leqq \infty .
\end{gathered}
$$

Applying Lemma 5 , we have that $A_{p} \cong A_{p}^{\prime}$ for all $p$.

Note that the converse to Theorem $\mathrm{A}$ is false. It is easy to construct nonisomorphic subgroups $G$ and $G^{\prime}$ of $Q \oplus Q$ which contain $Z \oplus Z$ with $G / Z \oplus Z \cong G^{\prime} \mid Z \oplus Z$. Since groups of rank two are determined up to isomorphism by their schemes, $S(G, X) \neq S\left(G^{\prime}, X^{\prime}\right)$ for any $X, X^{\prime}$. 
2. The intersection property. In this section we give a condition under which a group may be recovered from its scheme, $S(G, X)$.

Definition 2. The groups $A_{J}^{i}$ associated with $(G, X)$ are said to satisfy the intersection property iff $A_{J}^{i} \cap A_{J}^{2}=A_{J \cup J^{\prime}}^{i}$ for all $1 \leqq i \leqq n$ and $i \notin J \cup J^{\prime}$, where $J$ and $J^{\prime}$ are subsets of $\{1, \cdots, n\}$. In this case we will also say $(G, X)$ satisfies the intersection property.

REMARKS. It is immediate that if $G$ has rank $\leqq 2,(G, X)$ satisfies the intersection property for any maximal independent set $X \subseteq G$. If $\left(G_{i}, X_{i}\right)$ has the intersection property for $i=1,2, \cdots, n$, then $\left(\sum_{i=1}^{n} \oplus G_{i}\right.$, $\bigcup_{i=1}^{n} X_{i}^{\prime}$ ) has the intersection property, where $X_{i}^{\prime}$ denotes the natural embedding of $X_{i}$ into the direct sum. Let $v=\sum_{k=0}^{\infty} v_{k} p^{k}, w=\sum_{k=0}^{\infty} w_{k} p^{k}$ be $p$-adic units such that $1, v, w$ are rationally independent. Let $V_{0}=W_{0}=0$, $V_{i}=\sum_{k=0}^{2-1} v_{k} p^{k}, W_{i}=\sum_{k=0}^{i-1} w_{k} p^{k}$, for $i>1$. It can be shown using the methods of [1] that

$$
G=\left\{\left(r / p^{k}, r / p^{k} V_{k}+t, r / p^{k} W_{k}+l\right) \mid(r, p)=1, t, l \in Z\right\}
$$

is a strongly indecomposable rank 3 group; and it is easy to verify directly that $(G, X)$ has the intersection property with $X=\{(1,0,0),(0,1,0)$, $(0,0,1)\}$.

THEOREM B. Let $(G, X)$ satisfy the intersection property for $G$ a group of rank $n$ and $X=\left\{x_{1}, \cdots, x_{n}\right\}$ a maximal independent subset. Let $S(G, X)=\left\{A_{J}^{i}, \theta\right\}$. Then

$$
\bar{G}=\left\{\left(\alpha_{1}, \cdots, \alpha_{n}\right) \in Q^{n} \mid \alpha_{i} \in A^{i},\left(\alpha_{i}+A_{j}^{i}\right)^{\theta}=\alpha_{j}+A_{i}^{j}, i, j=1, \cdots, n\right\}
$$

is isomorphic to $G$ under the map $\left(\alpha_{1}, \cdots, \alpha_{n}\right) \rightarrow \sum_{i=1}^{n} \alpha_{i} x_{i}$.

Proof. We show that $\left(\alpha_{1}, \cdots, \alpha_{n}\right) \in \bar{G}$ iff $\sum_{i=1}^{n} \alpha_{i} x_{i} \in G$. Clearly, by definition of the maps $\theta, \sum_{i=1}^{n} \alpha_{i} x_{i} \in G$ implies $\left(\alpha_{1}, \cdots, \alpha_{n}\right) \in \bar{G}$. Let $\left(\alpha_{1}, \cdots, \alpha_{n}\right) \in \bar{G}$. Then $\alpha_{1} \in A^{1}$, so $g_{1}=\alpha_{1} x_{1}+\alpha_{2}^{\prime} x_{2}+\cdots+\alpha_{n}^{\prime} x_{n} \in G$ for some $\alpha_{2}^{\prime}, \cdots, \alpha_{n}^{\prime}$. But $\alpha_{2}^{\prime}+A_{1}^{2}=\left(\alpha_{1}+A_{2}^{1}\right)^{\theta}=\alpha_{2}+A_{1}^{2}$, so $\alpha_{2}-\alpha_{2}^{\prime} \in A_{1}^{2}$. Thus, there exists $g_{2}=\left(\alpha_{2}-\alpha_{2}^{\prime}\right) x_{2}+\alpha_{3}^{\prime \prime} x_{3}+\cdots+\alpha_{n}^{\prime \prime} x_{n} \in G$. Now $g_{1}+g_{2}=\alpha_{1} x_{1}+$ $\alpha_{2} x_{2}+\left(\alpha_{3}^{\prime}+\alpha_{3}^{\prime \prime}\right) x_{3}+\cdots+\left(\alpha_{n}^{\prime}+\alpha_{n}^{\prime \prime}\right) x_{n} \in G$. This yields $\alpha_{3}-\left(\alpha_{3}^{\prime}+\alpha_{3}^{\prime \prime}\right) \in A_{1}^{3} \cap$ $A_{2}^{3}$. Since $(G, X)$ satisfies the intersection property, we have $\alpha_{3}-\left(\alpha_{3}^{\prime}+\alpha_{3}^{\prime \prime}\right) \in$ $A_{12}^{3}$, and there exists $g_{3}=\left[\alpha_{3}-\left(\alpha_{3}^{\prime}+\alpha_{3}^{\prime \prime}\right)\right] x_{3}+\alpha_{4}^{\prime \prime \prime} x_{4}+\cdots+\alpha_{n}^{\prime \prime \prime} x_{n} \in G$. We continue in this way, obtaining $g_{1}, \cdots, g_{n}$, such that $\sum_{i=1}^{n} \alpha_{i} x_{i}=g_{1}+g_{2}+$ $\cdots+g_{n} \in G$.

Corollary. Let $(G, X)$ satisfy the intersection property, and let $S(G, X)=S\left(G^{\prime}, X^{\prime}\right)$. Then $G \cong G^{\prime}$.

Remark (AdDed IN PROOF). Theorem B and the above Corollary do not hold in general. There exist two nonisomorphic groups of rank 3 which have the same schemes with respect to the standard basis. 


\section{REFERENCES}

1. R. A. Beaumont and R. S. Pierce, Torsion-free rings, Illinois J. Math. 5 (1961), 61-98. MR 26 \#6212.

2. R. A. Beaumont and R. J. Wisner, Rings with additive group which is a torsion-free group of rank two, Acta Sci. Math. (Szeged) 20 (1959), 105-116. MR 21 \#5651.

Department of Mathematics, University of Connecticut, Storrs, Connecticut 06268 